\title{
Sensitivity improvement in two-center holographic recording
}

\author{
Ali Adibi, Karsten Buse, and Demetri Psaltis \\ Department of Electrical Engineering, California Institute of Technology, Pasadena, California 91125
}

Received December 16, 1999

\begin{abstract}
Persistent holograms are recorded with green light in $\mathrm{LiNbO}_{3}$ crystals doped with $\mathrm{Mn}$ and Fe. The recording sensitivity is 20 times better than that obtained by recording with red light. Partial loss of persistence is caused by using green light for recording. (c) 2000 Optical Society of America

OCIS code: $\quad 090.2900$.
\end{abstract}

Several holographic storage demonstrations in which iron-doped photorefractive lithium niobate $\left(\mathrm{LiNbO}_{3}: \mathrm{Fe}\right)$ was used were presented in the past few years. ${ }^{1,2}$ Erasure of the holograms during readout has been one of the major problems in the practical realization of holographic read-write memories. We recently proposed and demonstrated a new, two-center holographic recording method of solving this problem by use of doubly doped $\mathrm{LiNbO}_{3} .{ }^{3}$ Nondestructive readout was demonstrated in the initial experimental results, ${ }^{3}$ but low recording sensitivity was the main problem with the method. In this Letter we demonstrate that by using a shorter wavelength for the recording light we can increase the sensitivity by a large factor. The partial loss of persistence (nondestructive readout) caused by using light of shorter wavelength for holographic reading is acceptable for many practical systems.

We performed experiments with a congruently melting $x$-cut 0.85 -mm-thick $\mathrm{LiNbO}_{3}$ crystal doped with 0.075 -wt. $\% \mathrm{Fe}_{2} \mathrm{O}_{3}$ and 0.01-wt. \% MnO. Properly annealing the crystal ${ }^{4}$ yielded fully empty Fe traps and partially (more than 90\%) filled Mn traps before sensitization with UV light. A $100-W$ mercury lamp (wavelength, $404 \mathrm{~nm}$, unpolarized; intensity, $4 \mathrm{~mW} / \mathrm{cm}^{2}$, homogeneous) was used as the UV source. Recording was performed with two plane waves of coherent light with equal intensities and ordinary polarization interfering inside the crystal, with the UV light simultaneously illuminating the crystal. The angle between each beam and the normal the to crystal surface outside the crystal was $21^{\circ}$. The grating vector was aligned parallel to the $c$ axis of the sample. Readout of each hologram was performed by use of only one of the recording beams, with the UV beam blocked.

The basic idea of two-center holographic recording is to use the UV light to bring the electrons from $\mathrm{Mn}$ to $\mathrm{Fe}$ via the conduction band, use these electrons to record the hologram with red or green light, and eventually transfer the electrons from the Fe centers back to the Mn centers by use of red or green light. This process results in a hologram that is stored in Mn centers that persists against further red or green illumination.

Figures 1(a) and 1(b) show typical recording and readout curves for recording with red light (wavelength, $633 \mathrm{~nm}$ ) and green light (wavelength, $514 \mathrm{~nm}$ ), respectively, with simultaneous UV sensitization. In these experiments the intensity of each red beam was $300 \mathrm{~mW} / \mathrm{cm}^{2}$, and that of each green beam was $17 \mathrm{~mW} / \mathrm{cm}^{2}$. As Fig. 1 shows, the recording dynamics in both cases are similar, although recording with red light results in better persistence. The measure of recording speed, sensitivity $(S)$, is normally defined $a^{5}$

$$
S=\frac{\left.(\mathrm{d} \sqrt{\eta} / \mathrm{d} t)\right|_{t=0}}{I_{\operatorname{Rec}} L}
$$

In Eq. (1), $\eta, t, I_{\text {Rec }}$, and $L$ are diffraction efficiency (ratio of diffracted intensity to incident reading intensity), time, total recording intensity (sum of the intensities of the two recording beams), and crystal thickness, respectively. The units of sensitivity in Eq. (1) are in centimeters per joule. Although Eq. (1) is a good measure of sensitivity in normal holographic recording, it
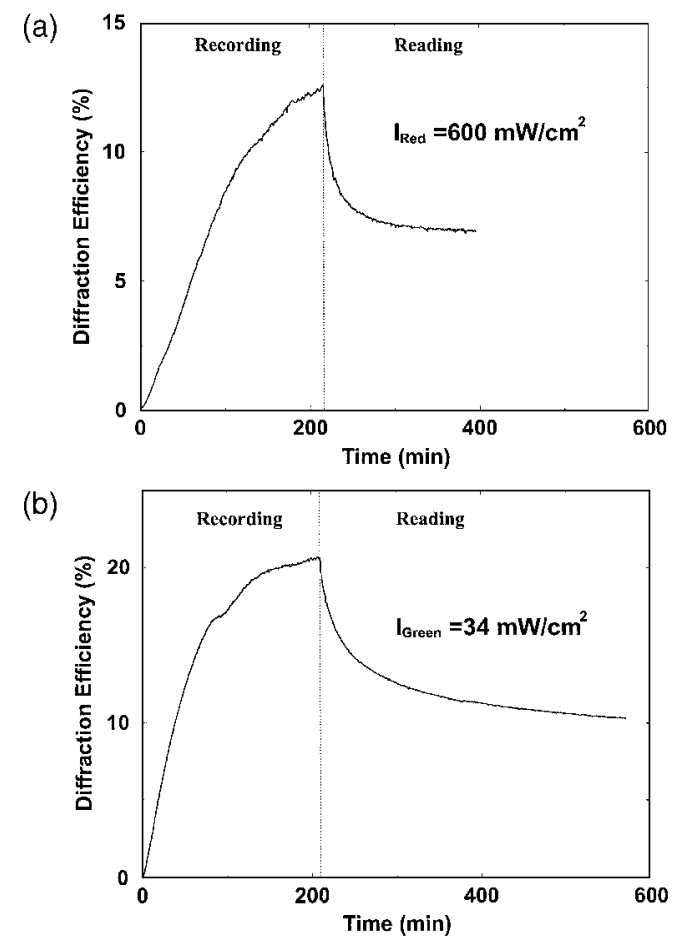

Fig. 1. Recording and readout curves for a plane-wave hologram in a $0.85-\mathrm{mm}$-thick $\mathrm{LiNbO}_{3}: \mathrm{Fe}: \mathrm{Mn}$ crystal. Recording was performed by simultaneous presence of a homogeneous UV beam and (a) two red beams or (b) two green beams. 
does not include the effect of partial erasure during readout in two-center holographic recording. We can modify Eq. (1) to obtain a better measure of sensitivity in two-center holographic recording:

$$
S^{\prime}=\beta S=\beta \frac{\left.(\mathrm{d} \sqrt{\eta} / \mathrm{d} t)\right|_{t=0}}{I_{\operatorname{Rec}} L},
$$

where $\beta$ is the ratio of $\sqrt{\eta}$ after sufficient readout to $\sqrt{\eta}$ at the end of recording (before any readout).

Typical sensitivities obtained in normal holographic recording in $\mathrm{LiNbO}_{3}: \mathrm{Fe}$ crystals in transmission geometry with ordinary polarization are $S \simeq 0.05-0.3 \mathrm{~cm} / \mathrm{J}$ for recording with blue light $(488 \mathrm{~nm})$ and $S \simeq 0.01-0.1 \mathrm{~cm} / \mathrm{J}$ for recording with red light $(633 \mathrm{~nm})$. Typical sensitivities obtained in two-center holographic recording in $\mathrm{LiNbO}_{3}: \mathrm{Fe}: \mathrm{Mn}$ crystals are $S^{\prime}=0.0033 \mathrm{~cm} / \mathrm{J}$ and $S^{\prime}=0.07 \mathrm{~cm} / \mathrm{J}$ with red (633-nm) and green (514-nm) light, respectively (Fig. 1). The main reason for the smaller sensitivity obtained in two-center holographic recording compared with that in normal recording is the recording mechanism. This difference can be explained by use of Fig. 2, which shows the energy-band diagrams for [Fig. 2(a)] a $\mathrm{LiNbO}_{3}$ : $\mathrm{Fe}$ crystal and [Fig. 2(b)] a $\mathrm{LiNbO}_{3}: \mathrm{Fe}: \mathrm{Mn}$ crystal. During recording in a $\mathrm{LiNbO}_{3}: \mathrm{Fe}$ crystal, electrons are excited from Fe traps to the conduction band by the recording light, move a short distance in the conduction band, and get trapped at Fe traps. This cycle of excitation, movement, and trapping is repeated while the hologram becomes stronger. Owing to the small mobility of electrons in the conduction band of $\mathrm{LiNbO}_{3}$, electrons need to go through this cycle many times for recording of strong holograms. As shown in Fig. 2(b), electrons are excited by UV light from either $\mathrm{Mn}^{2+}$ or $\mathrm{Fe}^{2+}$ into the conduction band, whereas red light excites electrons only from the shallower $\mathrm{Fe}^{2+}$ and green light excites electrons mostly from $\mathrm{Fe}$ traps. The conduction-band electrons can recombine with both centers, and thus UV illumination populates the $\mathrm{Fe}^{2+/ 3+}$ level partially, whereas red- or green-light illumination empties the Fe sites. During two-center holographic recording, the recording light excites electrons mainly from the Fe centers to the conduction band. These electrons are trapped at either Fe or Mn centers after moving a short distance in the conduction band. The electrons trapped in $\mathrm{Fe}$ centers can be used in the next cycle of excitation, movement, and trapping, whereas those trapped in Mn centers need to be transferred back to Fe centers (through the conduction band) by UV light before participating in another cycle. This requirement increases the average time of each cycle, resulting in a lower sensitivity than normal recording. This sensitivity loss can be reduced by use of higher UV intensities. Another reason for the smaller sensitivity in two-center recording is the partial erasure during readout represented by $\beta$ in Eq. (2). In the recording experiments shown in Fig. $1, \beta$ is $\sim 0.7$.

The sensitivity of holographic recording with red light is smaller than that of recording with blue or green light for both normal and two-center recording.
This smaller sensitivity is due to the position of $\mathrm{Fe}$ traps in the bandgap of $\mathrm{LiNbO}_{3}$. The absorption cross section of Fe traps at $633 \mathrm{~nm}$ is smaller than that at 514 or $488 \mathrm{~nm}$. The bulk photovoltaic coefficient of $\mathrm{Fe}$ traps has a similar variation with wavelength. The effect of different absorption cross section of Fe traps at different wavelengths can be better understood from the bleaching experiments shown in Fig. 3. First the crystal is sensitized by UV light for at least $1 \mathrm{~h}$, and then the sensitized crystal is illuminated with a green or a red beam and the transmitted beam is monitored. As electrons are transferred from $\mathrm{Fe}$ to $\mathrm{Mn}$ traps during bleaching, the absorption of the monitoring beam is reduced. As Fig. 3 shows, to obtain comparable bleaching speed with red and green light we need to have a much stronger red beam $\left(I_{\text {Red }}=300 \mathrm{~mW} / \mathrm{cm}^{2}\right.$, compared with $I_{\text {Green }}=17 \mathrm{~mW} / \mathrm{cm}^{2}$ ). Therefore we expect the sensitivity when green and UV light are used to be approximately $300 / 17 \simeq 18$ times better than the sensitivity when UV and red light are used. The small difference between this value and the actual measured value of a factor-of-20 sensitivity improvement probably is due to the role of additional factors such as the larger photovoltaic constant of the Fe traps at $514 \mathrm{~nm}$.

(a)

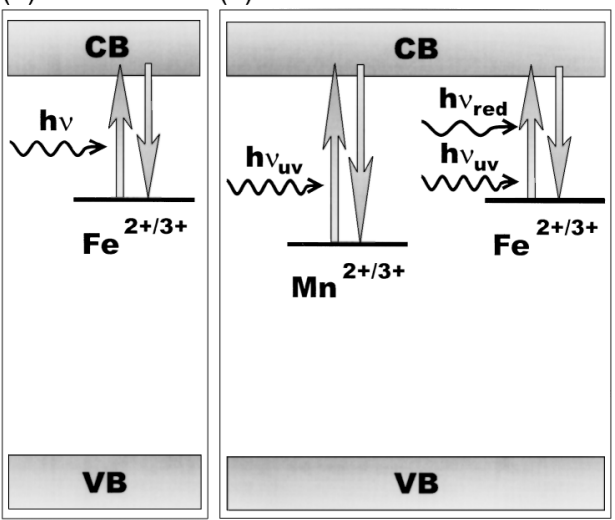

Fig. 2. Energy-band diagrams for a typical $\mathrm{LiNbO}_{3}$ crystal doped with (a) Fe or (b) Fe and Mn. CB, conduction band; VB, valence band.

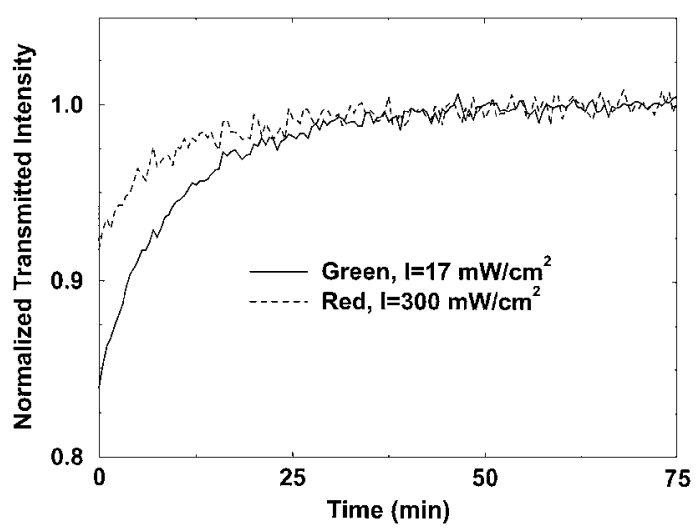

Fig. 3. Normalized intensity of transmitted light for bleaching experiments with a 0.85 -mm-thick, UVpreexposed $\mathrm{LiNbO}_{3}: \mathrm{Fe}: \mathrm{Mn}$ crystal. 
As Fig. 1 shows, one can improve the sensitivity of two-center holographic recording by a factor of 20 through the use of green instead of red recording light. However, using green light results in a partial loss of persistence, owing to the faster erasure of the hologram in $\mathrm{Mn}$ traps. One measure of the severity of this erasure is the number of times that we can read the entire memory before the diffraction efficiency of each hologram decreases to $50 \%$ of the original value. To calculate this number we first calculate the erasure-time constant by fitting the end part of the readout curve in Fig. 1(b) to an exponential function $[\sqrt{\eta}=C \exp (-t / \tau)]$. The curve-fitting results in $\tau=2 \times 10^{5} \mathrm{~s}$ for an erasing intensity of $I=$ $17 \mathrm{~mW} / \mathrm{cm}^{2}$. We then assume that the memory has a $1 \mathrm{~cm} \times 1 \mathrm{~cm} \times 1 \mathrm{~cm}$ crystal with $M / \#=10$, and 1000 holograms, each with a diffraction efficiency of $\eta=$ $10^{-4}$, are recorded. We also assume that each hologram contains a $1000 \times 1000$ two-dimensional data page (corresponding to $10 \mu \mathrm{m} \times 10 \mu \mathrm{m}$ pixels), resulting in a total capacity of 1 Gbit for the memory module, and that successful readout of each hologram requires the accumulation of 1000 photons per pixel. We also assume that both the recording and the readout intensities are $100 \mathrm{~mW} / \mathrm{cm}^{2}$. Therefore the readout time for each hologram is $t_{\text {read }}=1000 /[A \eta I /(h \nu)] \simeq 39 \mu \mathrm{s}$, where $A\left(=10^{-6} \mathrm{~cm}^{2}\right), \eta, I, h$, and $\nu$ are the pixel area, the diffraction efficiency of each hologram, readout intensity, Planck's constant, and the optical frequency of the readout beam, respectively. Therefore the readout time for the entire 1-Gbit memory is $1000 \times 39 \mu \mathrm{s}=$ $39 \mathrm{~ms}$. Since the erasure-time constant is inversely proportional to the reading intensity, we can calculate the erasure-time constant at $100 \mathrm{~mW} / \mathrm{cm}^{2}$ from the value that we measured at $17 \mathrm{~mW} / \mathrm{cm}^{2}$ to be $\tau=$ $(17 / 100) \times 2 \times 10^{5} \mathrm{~s}=3.4 \times 10^{4} \mathrm{~s}$. The total time that we can read the hologram before the diffraction efficiency $(\eta)$ decreases to $50 \%$ of its original value (or $\sqrt{\eta}$ decreases to $70 \%$ of its original value) is $t=\tau \ln (\sqrt{2})=$ $1.2 \times 10^{4} \mathrm{~s}$. Therefore we can read all the information in the memory module approximately 300,000 times before the diffraction efficiency decreases to $50 \%$ of its original value. Similar calculations for recording with UV and red result in the possibility of reading the entire memory $2 \times 10^{6}$ times for the same criteria.

When the diffraction efficiency of each hologram falls below some minimum value, we need to refresh the entire memory module by reading out and recording all holograms. To calculate the refreshing time of the memory as we described above, we assume inplane (or extraordinary) polarization for the recording beams to obtain $S^{\prime}=3 \times 0.07=0.21 \mathrm{~cm} / \mathrm{J}$, owing to the larger electro-optic coefficient of $\mathrm{LiNbO}_{3}$ for extraordinary polarization $\left(r_{33} \simeq 3 r_{13}\right)$. Using Eq. (1) and assuming that the recording dynamics are linear for small diffraction efficiencies, we can calculate the recording time of a hologram with a diffraction efficiency of $10^{-4}$ to be $0.5 \mathrm{~s}$ for a 1 -cm-thick crystal with a recording intensity of $100 \mathrm{~mW} / \mathrm{cm}^{2}$. To record 1000 holograms with equal diffraction efficiencies (each equal to $10^{-4}$ ) we need to use the recently proposed recording schedule for two-center holographic recording. ${ }^{6}$ Taking into account different recording times for the different holograms, and assuming the recording time of the last hologram to be $0.5 \mathrm{~s}$, we obtain a total recording time of $t \simeq 1000 \ln (1000) \times$ $0.5 \mathrm{~s} \simeq 3500 \mathrm{~s}$.

Although the focus of this Letter is on sensitivity improvement by use of green light, we also improve the dynamic range parameter, $M / \#,{ }^{7}$ by use of green light. This parameter can be inferred from Fig. 1 by comparison of the final diffraction efficiencies of recording with red light and green light. From Fig. 1, we can calculate $M / \# \simeq \sqrt{0.1}=0.32$ for recording with green light and $M / \# \simeq \sqrt{0.07}=0.26$. Using extraordinary polarization for the recording green beams, we obtain $M / \# \simeq 1$ for a 0.85 -mm-thick crystal. Using a 1-cm-thick sample, we expect to have a value of $M / \#$ of at least 10 , in agreement with the assumed value of $M / \#$ in previous calculations.

This work was supported by a Jet Propulsion Laboratory work order funded by the Defense Advanced Research Projects Agency/Information Technology Office and by U.S. Air Force Rome Lab Award F0060297C0049. A. Adibi's e-mail address is adibi@ sunoptics.caltech.edu.

\section{References}

1. F. H. Mok, Opt. Lett. 18, 915 (1993).

2. I. McMichael, W. Christian, D. Pletcher, T. Y. Chang, and J. H. Hong, Appl. Opt. 35, 2375 (1996).

3. K. Buse, A. Adibi, and D. Psaltis, Nature 393, 665 (1998).

4. A. Adibi, K. Buse, and D. Psaltis, Appl. Phys. Lett. 74, 3767 (1999).

5. P. Günter and J.-P. Huignard, eds., Photorefractive Materials and Their Applications I, Vol. 61 of Topics in Applied Physics (Springer-Verlag, Berlin, 1987).

6. A. Adibi, K. Buse, and D. Psaltis, Opt. Lett. 24, 652 (1999).

7. D. Psaltis, D. Brady, and K. Wagner, Appl. Opt. 27, 1752 (1988). 